\title{
Classification of Meteorites - Mössbauer Comparative Studies of Three Ordinary Chondrites Measured in Different Laboratories
}

\author{
P. Bogusz ${ }^{a, *}$, K. Brzózka ${ }^{b}$, B. Górka ${ }^{b}$, T. Szumiata $^{b}$, M. Wó́niaK $^{c}$ \\ AND J. GAŁAZZKA-FRIEDMAN ${ }^{a}$ \\ ${ }^{a}$ Warsaw University of Technology, Faculty of Physics, Koszykowa 75, 00-662 Warsaw, Poland \\ ${ }^{b}$ University of Technology and Humanities, Faculty of Mechanical Engineering, Department of Physics, \\ E. Stasieckiego 54, 26-600 Radom, Poland \\ ${ }^{c}$ Warsaw University, Faculty of Biology, Miecznikowa 1, 02-096 Warsaw, Poland
}

\begin{abstract}
Three meteorites — Bassikounou, Buzzard Coulee, and Zag — were studied with the use of the Mössbauer spectroscopy to assess how different experimental conditions, e.g. different statistics, velocity resolution and different ways of fitting the Mössbauer spectra could cause discrepancies regarding the percentages of spectral areas of main mineral phases (olivine, pyroxene, metallic phase and troilite) present in ordinary chondrites. The answer to this question is crucial for creation of a common database for a new method of classification of ordinary chondrites based on Mössbauer spectroscopy. Our results show that none of the above factors could significantly affect the percentage of spectral areas in the samples measured and the differences obtained with the use of those various methods of assessment were on the level of one percentage point. The final conclusion from this comparative study is that the results from different laboratories could be included to the same database.
\end{abstract}

DOI: 10.12693/APhysPolA.134.1070

PACS/topics: 96.30Za, 91.65.Sn, 75.50.Bb, 76.80.+y, 33.45.+x

\section{Introduction}

Meteorites are fragments of the planetoids, which gained the surface of the Earth after turbulent journey through the terrestrial atmosphere. Also lunar and Martian meteorites, which were probably ejected from the surface of Moon and Mars, are being found. Meteorites, which were collected soon after witnessed fall are called "falls". Meteorites, which were found later are called "finds". About eighty percent of falls are ordinary chondrites. There are three types of the ordinary chondrites: type $\mathrm{H}$ (high amount of iron), type L (low amount of iron), type LL (low amount of iron, low amount of metal).

Standard classification of ordinary chondrites is based on laborious mineralogical determination of the ratio of the amount of fayalite (present in olivines) to the amount of ferrosilite (present in orthopyroxenes). The Mössbauer spectra of the nonweathered ordinary chondrites are composed of two sextets from kamacite ( $\mathrm{FeNi}$ alloy with about $10 \%$ of $\mathrm{Ni}$ ) and troilite $(\mathrm{FeS})$ and two doublets from olivines $(\mathrm{Fe}, \mathrm{Mg})_{2} \mathrm{SiO}_{4}$ and pyroxenes $(\mathrm{Fe}$, $\mathrm{Mg})_{2} \mathrm{SiO}_{3}$. Kamacite and troilite can be created only in cosmic conditions.

In early 2000 Verma and his group started works on the application of the Mössbauer spectroscopy to the classification of ordinary chondrites [1-3]. This group decided to use 2D plots of spectral area of olivine vs. spectral area of pyroxene, spectral area of metallic phases

*corresponding author vs. spectral area of silicate phases, and so on. These plots were created in majority on the base of their own results. They were able to classify the meteorite ItawBhopij as ordinary chondrite type L. Classification was based on the determination of the position of unknown meteorite in cited plots. This determination was not fully objective. Regions typical for different types were determined arbitrary. These studies were continued by groups from Ekaterinburg and Canberra. The Ekaterinburg group [4] created its own base of results of Mössbauer data. The Canberra group [5] was using Verma's data base. Our preliminary results of the application of the Mössbauer spectroscopy to classification of ordinary chondrites were published in 2017 [6]. Our data base was composed from our own results and outcomes taken from the literature.

The goal of our present paper is testing the procedure of including, to one data base, the Mössbauer results of the percentages of spectral areas of four main mineral phases present in ordinary chondrites, obtained in different laboratories. This work was initiated by doubts of one of the Mössbauer specialist (unknown reviewer of our manuscript submitted to one of the astronomical journals). She/He wrote: "The authors used for their analysis Mössbauer parameters obtained by different researches using Mössbauer spectrometers with different quality and using different spectra fits. This is not correct because the relative areas of spectral components depend on several factors." To disperse these doubts, the samples of 3 ordinary chondrites were measured in two different laboratories in different experimental conditions and different ways of fitting. 


\section{Material and methods}

The sample of three ordinary chondrites type $\mathrm{H}$ : Bassikounou, Buzzard Coulee, Zag (Fig. 1) were investigated. All come from recent falls, so their specimens are not strongly weathered. Detailed data on these meteorites are as follows.

Bassikounou - ordinary chondrite type H5. Fall in October 2006 in Mauritania. Many fresh specimens with a total weight of about $29 \mathrm{~kg}$ were found.

Buzzard Coulee - ordinary chondrite type H4. Fall in November 2008 in Canada. About $41 \mathrm{~kg}$ of specimens were collected.

Zag — ordinary chondrite type H3-6. Fall in August 1998 in Western Sahara. Specimens are finding to this day. In total over $175 \mathrm{~kg}$ were collected.

The specimens of bulk meteorites were crushed and ground in alcohol into fine powder in an agate mortar. Finally, the specimens were loaded into plastic holders. The diameter of the active area of the specimen was $14 \mathrm{~mm}$.

The Mössbauer measurements were carried out in Magnetic Materials Laboratory belonging to Department of Physics of Faculty of Mechanical Engineering in University of Technology and Humanities in Radom. Room temperature transmission spectra were collected using ${ }^{57} \mathrm{Co} / \mathrm{Rh}$ source of gamma radiation (with activity $0.7 \mathrm{GBq}$ ) and spectrometer POLON, with vertical geometry of the radiation beam. A constant acceleration mode of the source motion was employed in order to modify the radiation energy. A proportional counter was used to detect radiation transmitted through the absorber and automatic system counted them in correlation with the source velocity with resolution 1024 channels. The number of counts was from 6.5 million to 9 million per channel (in a folded spectrum).

The Mössbauer spectra were analyzed by means of program NORMOS, based on least squares fitting of Lorentzian lines. For the component attributed to troilite, full Hamiltonian procedure was employed. Criteria of the best fit included both value of $\chi^{2}$ and physical meaning of fitted parameters. Moreover, the shape of differential spectrum yielded important hints concerning the number and the kind of subspectra and the values of their hyperfine parameters. The Zeeman sextets, related to magnetic phases, were fitted assuming the line area ratio $A_{1,6}: A_{2,5}: A_{3,4}=3: 2: 1$ (where $A_{1,6}$ denotes spectral area of line 1 or 6 in a sextet, etc.) and an equal line width within a sextet. The analysis allowed us to determine both hyperfine parameters of individual components and relative contribution of each of them, which enables to evaluate proportions between iron-bearing phases.

In order to exclude any artefacts originated from the method of fitting the spectra, a comparative fitting was carried out by means of PolMöss software, which utilizes parallel gradient and evolutionary optimization algorithms implemented in Solver extension for MS Excel. In general, PolMöss package offers the Voigt profile form of subspectra as a result of Lorentzian base-line convolution with Gaussian distributions of hyperfine parameters.
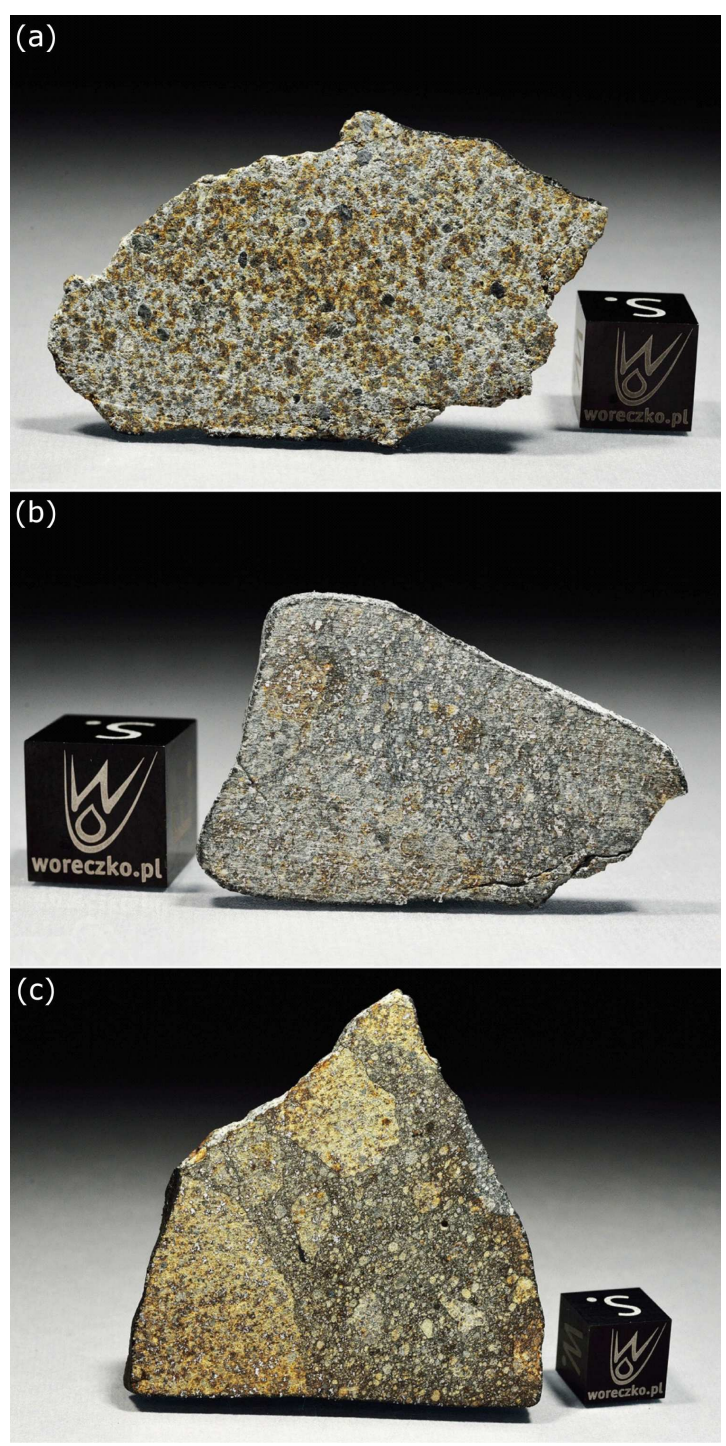

Fig. 1. Meteorites: Bassikounou (a), Buzzard Coulee (b), and Zag (c). The scale cube $1 \mathrm{~cm}$ (collection: Meteorites Collection Jan Woreczko \& Wadi; photo: Jan Woreczko).

Nevertheless in our case of very narrow lines corresponding to bulk, crystalline minerals, just simple Lorentzian profile was sufficient. PolMöss program was effectively utilized for the Mössbauer spectra analysis of dusts from high-ways, fly ashes from power plants and heat plants as well as for thin magnetic Fe-Ga films [7].

The Mössbauer measurements were carried out also in the Mössbauer Laboratory located in Faculty of Physics in Warsaw University of Technology. The Mössbauer measurements ${ }^{57} \mathrm{Fe} / \mathrm{Rh}$ were done at room temperature with a conventional spectrometer which belongs to „Elektronika jądrowa" of Wacław Musiałwith a horizontal geometry of the radiation beam and the source ${ }^{57} \mathrm{Co} / \mathrm{Rh}$ (activity $3.7 \mathrm{GBq}$ ). The gamma rays were detected by a proportional counter (refurbished Ar-izo-pentan, operating voltage $1540 \mathrm{~V}$ ). The controller-drive was the source of the triangular waveform. 
The Mössbauer measurements were carried out at room temperature in 512 channels. The spectrum was folded (to 256 channels) with the Recoil program based on a calibration spectrum. The velocity scales were calibrated with an iron foil absorber $\alpha$-Fe with a thickness of $0.05 \mathrm{~mm}$. The number of counts in the channel was about 3 million.

The fitting procedure was performed using the "Full Static Hamiltonian" analysis in the Recoil programme and then, using the option "Powder Crystal Site" the ratio of intensity of lines in a sextet was fixed as 3:2:1.

\section{Results and discussion}

The Mössbauer spectra obtained in Radom are presented in Figs. 2-4. Qualitatively all the spectra have similar character but they vary in respect of quantitative parameters (Table I). Three Zeeman sextets and three doublets were used to fit every spectrum. A doublet with central shift $\delta=(1.14-1.15) \mathrm{mm} / \mathrm{s}$ and quadrupole splitting $\Delta=(2.95-2.96) \mathrm{mm} / \mathrm{s}$, attributed to olivine, dominates in all the spectra. The second doublet related to pyroxene is also noticeable: its parameters equal: $\delta$ $=(1.14-1.15) \mathrm{mm} / \mathrm{s}$ and $\Delta=2.10 \mathrm{~mm} / \mathrm{s}$. The third doublet is clearly wider and much smaller, its hyperfine parameter values suggest that this component is related to $\mathrm{Fe}^{3+}$ ions.
A sextet with hyperfine magnetic field about $30.8 \mathrm{~T}$ and specific asymmetry is present in all the spectra in an amount approximately $17 \%$. It is related to troilite. There are two sextets of similar parameters in all the spectra: both with isomer shift and quadrupole shift near zero and hyperfine magnetic field about $B=34.1 \mathrm{~T}$ and $B=33.0 \mathrm{~T}$ for the first sextet and the second one, respectively. We attribute them to different positions of iron atoms in iron metallic phase in the form of $\mathrm{Fe}(\mathrm{Ni})$ or $\mathrm{Fe}(\mathrm{Ni}, \mathrm{Co})$ alloys.

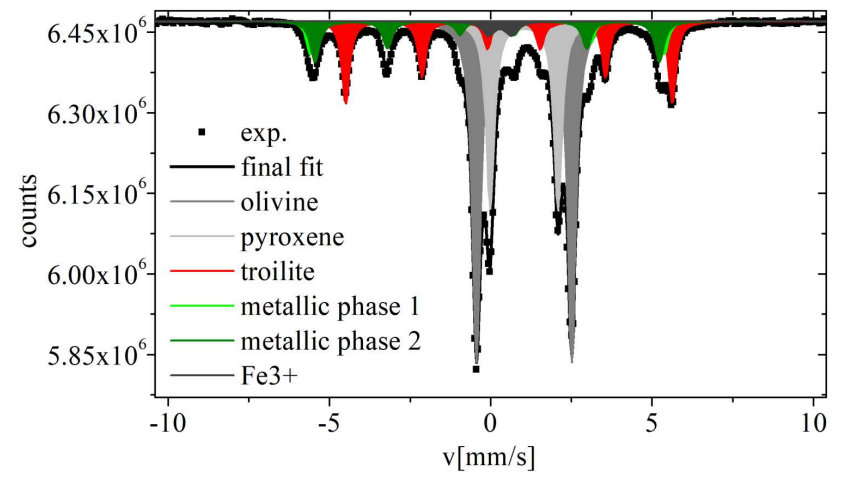

Fig. 2. Mössbauer spectrum of the Bassikounou meteorite obtained in Radom.

TABLE I

Hyperfine interactions parameters obtained from the best fit to the experimental Mössbauer spectra of meteorites Bassikounou, Buzzard Coulee, Zag (spectra collected in Radom, program Normos). IS - isomer shift, $B$ - internal magnetic field, $e^{2} q Q / 2$ - quadrupole interaction parameter, $\Theta$ - angle between direction of magnetic field and the main axis of the electric field gradient, $w$ - HWHM (half width at half maximum). Experimental uncertainties for fitted parameters are as follows: for $I S-0.01 \mathrm{~mm} / \mathrm{s}$, for $Q S-0.02 \mathrm{~mm} / \mathrm{s}$, for $B-0.2 \mathrm{~T}$, for $w-0.03 \mathrm{~mm} / \mathrm{s}$.

\begin{tabular}{|c|c|c|c|c|c|c|}
\hline Meteorite & Mineral phase & $I S[\mathrm{~mm} / \mathrm{s}]$ & $B[\mathrm{~T}]$ & $\overline{e^{2} q Q / 2[\mathrm{~mm} / \mathrm{s}]}$ & $\Theta\left[^{\circ}\right]$ & $w[\mathrm{~mm} / \mathrm{s}]$ \\
\hline \multirow{6}{*}{ Bassikounou } & olivine & 1.14 & - & 2.96 & - & 0.15 \\
\hline & pyroxene & 1.15 & - & 2.10 & - & 0.15 \\
\hline & troilite & 0.75 & 30.8 & 1.03 & 61.3 & 0.14 \\
\hline & metallic phase 1 & 0.02 & 34.1 & -0.04 & - & 0.14 \\
\hline & metallic phase 2 & 0.00 & 33.1 & 0.02 & - & 0.17 \\
\hline & $\mathrm{Fe} 3+$ & 0.40 & - & 0.71 & - & 0.23 \\
\hline \multirow{6}{*}{ Buzzard Coulee } & olivine & 1.15 & - & 2.95 & - & 0.15 \\
\hline & pyroxene & 1.14 & - & 2.10 & - & 0.15 \\
\hline & troilite & 0.75 & 30.8 & 1.03 & 61.1 & 0.14 \\
\hline & metallic phase 1 & 0.01 & 34.0 & -0.02 & - & 0.14 \\
\hline & metallic phase 2 & 0.01 & 32.9 & -0.01 & - & 0.15 \\
\hline & $\mathrm{Fe} 3+$ & 0.41 & - & 0.76 & - & 0.26 \\
\hline \multirow{6}{*}{ Zag } & olivine & 1.15 & - & 2.95 & - & 0.15 \\
\hline & pyroxene & 1.15 & - & 2.10 & - & 0.15 \\
\hline & troilite & 0.75 & 30.8 & 1.01 & 61.4 & 0.14 \\
\hline & metallic phase 1 & 0.02 & 34.2 & -0.03 & - & 0.15 \\
\hline & metallic phase 2 & 0.01 & 33.1 & 0.00 & - & 0.17 \\
\hline & $\mathrm{Fe} 3+$ & 0.39 & - & 0.73 & - & 0.25 \\
\hline
\end{tabular}




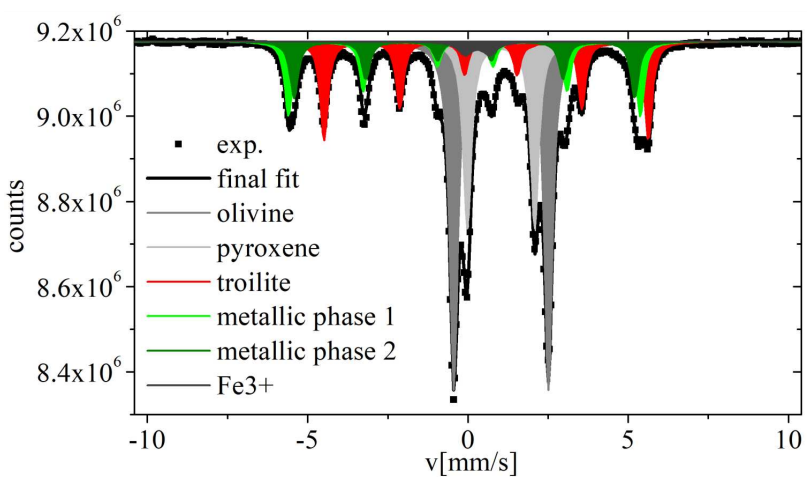

Fig. 3. Mössbauer spectrum of the Buzzard Coulee meteorite obtained in Radom.

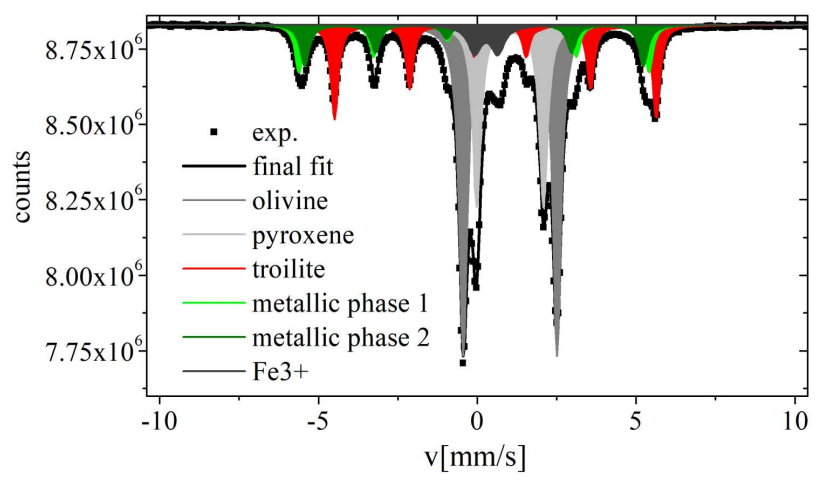

Fig. 4. Mössbauer spectrum of the Zag meteorite obtained in Radom.

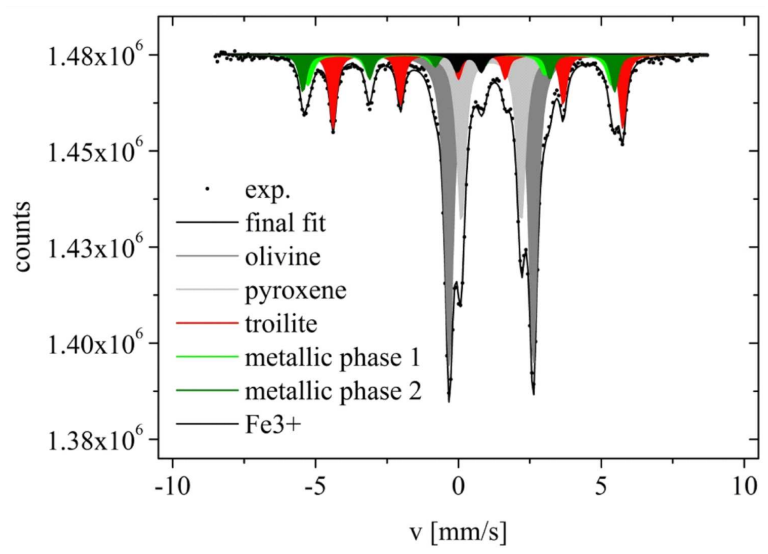

Fig. 5. Mössbauer spectrum of the Bassikounou meteorite obtained in Warsaw.

Mössbauer spectra of three ordinary chondrites type $\mathrm{H}$ obtained in Warsaw are shown in Fig. 5 (meteorite Bassikounou), in Fig. 6 (meteorite Buzzard Coulee) and in Fig. 7 (meteorite Zag). They are composed of 2 doublets (associated with olivine and pyroxene ) and 3 sextets (associated with two metallic phases and troilite). In each spectrum ferric doublet is also present. Identification of mineral phases was based on hyperfine interactions parameters obtained by fitting theoretical curves

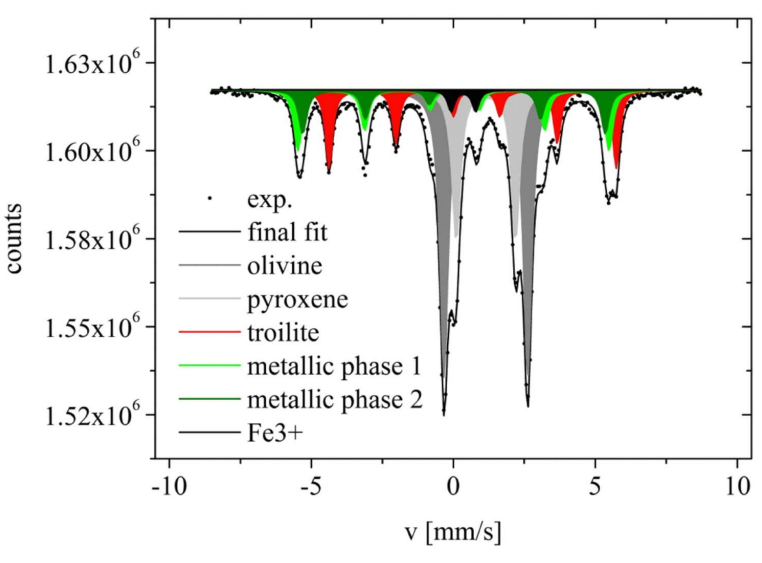

Fig. 6. Mössbauer spectrum of the Buzzard Coulee meteorite obtained in Warsaw.

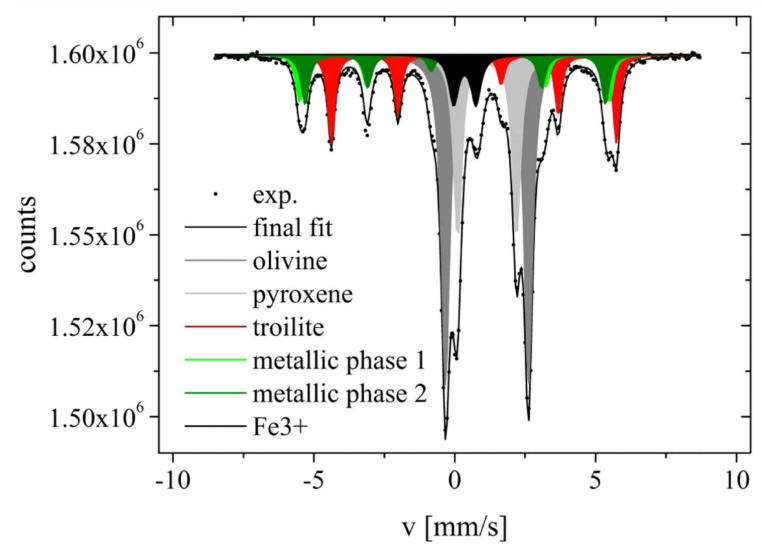

Fig. 7. Mössbauer spectrum of the Zag meteorite obtained in Warsaw.

to the experimental points (see Table II). The parameters obtained in Warsaw and in Radom are very similar. The only significant difference are broadened line widths present in Warsaw spectra. Urban situation of WUT campus (intensive traffic) is the reason of these broadening.

All hyperfine interactions parameters for olivine, pyroxene, troilite and kamacite are in the range of literature data [3]. The values of $Q S$ for troilite are different, because in the literature troilite spectra were analyzed assuming that theta angle (angle between direction of magnetic field and the main axis of the electric field gradient) is equal to zero. It is not true. Assuming that theta angle is not equal to zero, we have got different, correct values of $Q S$. Doublet of ferric iron with IS $\approx 0.4 \mathrm{~mm} / \mathrm{s}$ is associated with nanoparticles of iron oxides which are the results of the beginning of weathering process.

Table III contains values of spectral areas of four mineral phases (and $\mathrm{Fe} 3+$ ) present in the meteorites Bassikounou, Buzzard Coulee, Zag (spectra collected in Radom and Warsaw). In column I results obtained in Radom (spectra fitted with the use of program Normos and assumption that theta angle is different than zero), are presented. In column II also results obtained in Radom 
TABLE II

Hyperfine interactions parameters obtained from the best fit to the experimental Mössbauer spectra of meteorites Bassikounou, Buzzard Coulee, Zag (spectra collected in Warsaw, program Recoil). IS - isomer shift, $B$ - internal magnetic field, $e^{2} q Q / 2$ - quadrupole interaction parameter, $\Theta$ - angle between direction of magnetic field and the main axis of the electric field gradient, $w$ - HWHM (half width at half maximum). Experimental uncertainties for fitted parameters are the following: for $I S-0.01 \mathrm{~mm} / \mathrm{s}$, for $Q S-0.02 \mathrm{~mm} / \mathrm{s}$, for $B-0.2 \mathrm{~T}$, for $w-0.03 \mathrm{~mm} / \mathrm{s}$.

\begin{tabular}{c|l|c|c|c|c|c}
\hline \hline Meteorite & Mineral phase & $I S[\mathrm{~mm} / \mathrm{s}]$ & $B[\mathrm{~T}]$ & $e^{2} q Q / 2[\mathrm{~mm} / \mathrm{s}]$ & $\Theta\left[{ }^{\circ}\right]$ & $w[\mathrm{~mm} / \mathrm{s}]$ \\
\hline \multirow{5}{*}{ Bassikounou } & olivine & 1.14 & - & 2.95 & - & 0.18 \\
& pyroxene & 1.15 & - & 2.10 & - & 0.17 \\
& troilite & 0.75 & 30.9 & 1.01 & 61.1 & 0.14 \\
& metallic phase 1 & 0.03 & 33.8 & -0.04 & - & 0.15 \\
& metallic phase 2 & -0.01 & 33.0 & 0.10 & - & 0.20 \\
& Fe3+ & 0.38 & - & 0.85 & - & 0.17 \\
\hline \multirow{5}{*}{ Buzzard Coulee } & olivine & 1.15 & - & 2.95 & - & 0.18 \\
& pyroxene & 1.15 & - & 2.09 & - & 0.18 \\
& troilite & 0.75 & 30.8 & 1.01 & 61.1 & 0.15 \\
& metallic phase 1 & 0.03 & 34.0 & -0.05 & - & 0.17 \\
& metallic phase 2 & 0.01 & 33.0 & 0.03 & - & 0.17 \\
& Fe3+ & 0.36 & - & 0.87 & - & 0.15 \\
\hline \multirow{5}{*}{ Zag } & olivine & 1.14 & - & 2.95 & - & 0.18 \\
& pyroxene & 1.15 & - & 2.09 & - & 0.18 \\
& troilite & 0.75 & 30.9 & 1.01 & 61.5 & 0.15 \\
& metallic phase 1 & 0.03 & 34.1 & -0.06 & - & 0.17
\end{tabular}

TABLE III

Values of spectral areas of four mineral phases (and $\mathrm{Fe}^{3+}$ ) present in the meteorites Bassikounou, Buzzard Coulee, Zag.

\begin{tabular}{|c|c|c|c|c|c|c|c|}
\hline \multicolumn{2}{|c|}{ Spectra collected in } & \multirow{2}{*}{$\begin{array}{c}\begin{array}{c}\text { Radom, } \\
\text { program } \\
\text { Normos }\end{array} \\
\text { I } \\
\end{array}$} & \multirow{2}{*}{$\begin{array}{c}\text { Radom, } \\
\text { program } \\
\text { PolMöss } \\
\text { II }\end{array}$} & \multirow{2}{*}{$\begin{array}{c}\text { Warsaw, } \\
\text { program } \\
\text { Recoil }\end{array}$} & \multirow{2}{*}{$\begin{array}{c}\text { Warsaw, } \\
\text { program } \\
\text { Recoil }\end{array}$} & \multirow{2}{*}{$\begin{array}{c}\begin{array}{c}\text { Difference } \\
\text { I-III }\end{array} \\
\mathrm{V} \\
\end{array}$} & \multirow{2}{*}{$\begin{array}{c}\text { Difference } \\
\text { II-IV } \\
\text { VI }\end{array}$} \\
\hline & & & & & & & \\
\hline Meteorite & Mineral phase & $\begin{array}{c}\text { A with } \\
\Theta \neq 0[\%]\end{array}$ & $\begin{array}{c}\text { A with } \\
\Theta=0[\%]\end{array}$ & $\begin{array}{c}\text { A with } \\
\Theta \neq 0[\%]\end{array}$ & $\begin{array}{c}\text { A with } \\
\Theta=0[\%]\end{array}$ & & \\
\hline \multirow{5}{*}{ Bassikounou } & olivine & 39.8 & 39.8 & 41.3 & 41.3 & -1.5 & -1.6 \\
\hline & pyroxene & 22.0 & 22.2 & 22.0 & 22.2 & 0 & 0 \\
\hline & troilite & 16.6 & 16.7 & 16.3 & 16.7 & 0.3 & 0 \\
\hline & metallic phases & 17.9 & 17.9 & 18.1 & 17.7 & -0.2 & 0.2 \\
\hline & $\mathrm{Fe}_{3+}$ & 3.6 & 3.4 & 2.3 & 2.1 & 1.3 & 1.3 \\
\hline \multirow{5}{*}{ Buzzard Coulee } & olivine & 35.0 & 35.1 & 36.3 & 36.3 & -1.3 & -1.2 \\
\hline & pyroxene & 19.1 & 19.2 & 19.0 & 19.1 & 0.1 & 0.1 \\
\hline & troilite & 17.8 & 17.6 & 17.0 & 17.2 & 0.8 & 0.4 \\
\hline & metallic phases & 24.3 & 24.6 & 25.4 & 25.3 & -1.1 & -0.7 \\
\hline & $\mathrm{Fe}_{3+}$ & 3.8 & 3.5 & 2.3 & 2.1 & 1.5 & 1.4 \\
\hline \multirow{5}{*}{ Zag } & olivine & 35.7 & 35.8 & 36.4 & 37.0 & -0.7 & -1.2 \\
\hline & pyroxene & 20.0 & 20.1 & 20.0 & 20.3 & 0 & -0.2 \\
\hline & troilite & 16.8 & 17.0 & 16.7 & 17.3 & 0.1 & -0.3 \\
\hline & metallic phases & 19.8 & 19.5 & 20.5 & 19.5 & -0.7 & 0 \\
\hline & $\mathrm{Fe}_{3+}$ & 7.9 & 7.6 & 6.3 & 5.9 & 1.6 & 1.7 \\
\hline
\end{tabular}

are shown, but spectra were fitted with the use of program PolMöss with the assumption that theta angle is equal to zero. In column III and IV results obtained in Warsaw (spectra fitted with the use of program Recoil) are presented: in column III results obtained with the assumption that theta angle is different than zero and in column IV results obtained with the assumption that theta angle is equal zero. Analysis of the obtained results shows that two different methods of the fitting of the subspectrum of troilite (one with the assumption, 
that $\Theta \neq 0$, and the second, that $\Theta=0$ ) do not have any influence on the values of the percentages of spectral areas. Observed discrepancies are on the level of the differences which occur when we fit the spectra once again using different initial parameters.

In columns V and VI differences of the results obtained in Radom and in Warsaw are shown (fitting with the assumption that that $\Theta \neq 0$ (column V) and $\Theta=0$ (column VI), respectively). Values of these differences comprise in the range typical for differences present at new fitting $(-1.6,+1.7)$. We did not notice any systematics, which could support the hypothesis that instrumental factor or mode of fitting can influence the percentage of spectral areas.

Figure 8 shows the results of our present study (in blue) presented against a background of relationship between percentage of spectra area from olivines (ol) vs. percentage of spectral area from metallic phase (met) obtained for ordinary chondrites type LL, L and H. This plot was created with the use of our database published in 2017 [6].

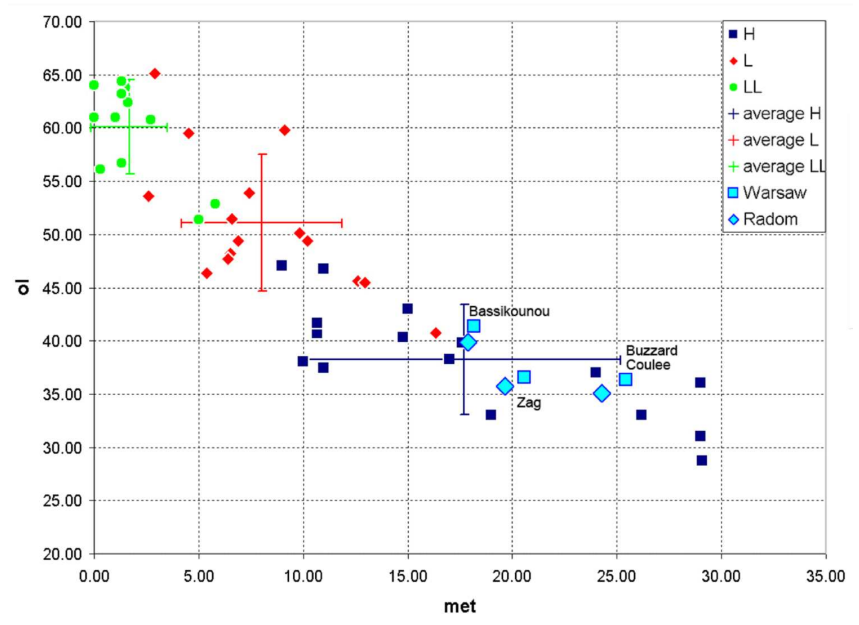

Fig. 8. The relationship between percentage of spectra area of olivines (ol) vs. percentage of spectral area of metallic phase.

At that time our database was composed of 13 Mössbauer spectra of ordinary chondrites type LL (10 our own data, 3 spectra taken from the literature), 15 Mössbauer spectra of ordinary chondrites type L (6 our own data, 10 spectra taken from the literature), and 16 Mössbauer spectra of ordinary chondrites type $\mathrm{H}$ (6 our own data, 10 spectra taken from the literature). The Mössbauer spectra taken from the literature were obtained in following laboratories: Indian Institute of Technology, Kanpur [2], Slovak University of Technology, Bratislava [8], Ural State Technical University, Ekaterinburg (Russia) [4], University of Parma (Italy) [9], Mössbauer Effect Data Center, University of North Carolina at Asheville (USA) [10], University of Uppsala (Sweden) [11].
Big dispersion of the points representing different meteorites do not witness a big uncertainty of the results obtained in different laboratories. This dispersion is caused by inhomogeneity of the material which is present in all ordinary chondrites.

The results obtained in present work (and marked by blue points in Fig. 8) are additional argument for the rightness of the above statement.

\section{Conclusions}

Comparing the results of Mössbauer spectroscopy investigation performed in two laboratories and at various conditions, we did not notice any systematics, which could support the hypothesis that instrumental factors or mode of fitting can influence the percentage of spectral areas. Differences in the percentages of spectral areas of the individual mineral phases were at the level of one percentage point. Therefore, there is no reason not to include results from different laboratories to the same database.

\section{Acknowledgments}

The samples of meteorites for this study and for construction of Fig. 8 were generously supplied by Marcin Cimała, Ansgar Greshake, Mike Gilmer, Tomasz Jakubowski, Kazimierz Mazurek, Jarosław Morys, Krzysztof Socha, Robert Verish, Łukasz Karwowski, and Jan Woreczko.

\section{References}

[1] B.S. Paliwal, R.P. Tripathi, H.C. Verma, S.K. Sharma, Meteorit. Planet. Sci. $\quad 35,639$ (2000).

[2] H.C. Verma, A. Rawat, B.S. Paliwal, R.P. Tripathi, Hyperfine Interact. 142, 643 (2002).

[3] H.C. Verma, K. Jee, R.P. Tripathi, Meteorit. Planet. Sci. 38, 963 (2003).

[4] M.I. Oshtrakh, E.V. Petrova, V.I. Grokhovsky, V.A. Semionkin, Meteorit. Planet. Sci. 43, 941 (2008).

[5] N.N. Elewa, J.M. Cadogan, Hyperfine Interact. 238 4 (2017).

[6] J. Gałązka-Friedman, M. Woźniak, P. Duda, P. Rzepecka, M. Jakubowska, Ł. Karwowski, Hyperfine Interact. 238, 67 (2017).

[7] A. Javed, T. Szumiata, N.A. Morley, M.R.J. Gibbs, Acta Mater. 58, 4003 (2010).

[8] J. Lipka, J. Sitek, J. Dekan, J. Degmová, V. Porubčan, Hyperfine Interact. 218, 107 (2013).

[9] I. Ortalli, G. Pedrazzi, Hyperfine Interact. 57, 2275 (1990).

[10] Y. Zhang, J.G. Stevens, Y. Li, Z. Li, Hyperfine Interact. 91, 547 (1994).

[11] Y.A. Abdu, T. Ericsson, Meteorit. Planet. Sci. 32, $373(1997)$. 